\title{
The New Doctrine of Acute Pneumonia-the Key to Solve the Problem Igor Klepikov
}

Pediatric Surgeon, 2116 27th St. NE Renton, WA, USA

Pneumonia was described 2,500 years ago by Hippocrates, the father of medicine [1]. However, acute pneumonia (AP) remain an unresolved problem of modern medicine. A lot of money and effort is expended on solving problem of AP. In this connection, it is recalled that World Health Organization has spent 39 billions of dollars in 2010-2015 years to solve this problem [2]. And where are the results? The evaluation of the current situation in this section of clinical medicine looks hopeless, and her improvement is very uncertain.

Pneumonia is a leading cause of hospitalization among children in the United States, with medical costs estimated at almost $\$ 1$ billion in 2009. Despite this large burden of disease, critical gaps remain in our knowledge about pneumonia in children [3].

Pediatric pleural empyema has increased substantially over the past 20 years and reasons for this rise remain not fully explained [4].

It is necessary to consider the fact that a detailed evaluation of results of treatment of AP is, as a rule, in conditions of the elite health systems. The status of this problem in developing countries is not always reflected in the available literature. Cumulative results on the problem in these parts of the world indicate high morbidity and mortality in AP.

To great regret, practical medicine tries to solve the problem of AP too simple (even primitive) way. Triumphal start of the era of antibiotics gave rise to an atmosphere of euphoria. However, hopes for the effectiveness of one of the universal remedy remain the main focus of the strategy and tactics in AP for the past several decades $[5,6]$. Antibiotics are considered such a panacea [7]. Indeed, the discovery of antibiotics has been one of the most outstanding achievements of 20th century medicine. This fact cannot be doubted. Antibiotics remain the main mode of treatment of $\mathrm{AP}$, despite reducing its effectiveness in comparison with the initial period of use. The essence of the existing dilemma is as follows: on the one hand, the antibiotics alone is enough for a large part of patients with AP and their bodies are themselves coping with the disease. On the other hand, in developed (!) countries from $9.5 \%$ to $42 \%$ of patients with pneumonia are received in the hospital due to the ineffectiveness of primary treatment [8]. However, the transfer of patients to hospital does not improve the results of treatment. Parapneumonic effusions or empyema affect 2 to 12 percent of children with pneumonia, and up to 28 percent of those requiring hospitalization [9].

The unique role of the lungs in the body is well known, including non-respiratory functions. Also it is well known that the reaction of everyone to certain stimulus has individual character. Therefore, the speed of development of inflammation, the intensity of occurring disorders and the body's ability to adapt have a huge range of options. Reflex influence of inflammation in the lung on the small circle of blood circulation causes the inevitable restructuring of the systemic circulation. The characteristics and course of action of therapeutic activities allow both inhibition of the inflammatory process and its stimulation. Therefore it is very important to have an understanding of the pathogenesis of the disease and the impact of medical procedures on its links. And should recognize the obvious fact that even more

\section{Publication History:}

Received: May 01, 2017

Accepted: May 29, 2017

Published: May 31, 2017

\section{Keywords:}

Acute pneumonia, Practical medicine, Prevention, Inflammation

effective antibiotics will not allow to guarantee the prevention of the complications of AP. The key solution to this problem is hidden in the pathogenesis of the disease.

To date, medical science has accumulated a huge amount of information on various aspects related to the AP. Many medical disciplines have made great strides in the study of the role of the lungs in the body. The features of nonspecific inflammation are thoroughly and comprehensively studied. However, all of these achievements, as a rule, do not go beyond their own discipline. Clinical approaches to AP formed without considering important information, well-known pathophysiology, anatomy, etc. If practical doctors are aware of these issues, this does not affect the views on the nature of the disease and subsequent tactics. If we do not attach importance to certain biological rules and laws, this does not mean that they can be bypassed. These laws will operate regardless of our desires, isn't it? It is impossible to study any subject or phenomenon. if we consider this object with a fixed position. It is necessary to evaluate the possible maximum of its characteristics. At the same time, when there are many disparate facts relating to the object under study, it is very difficult to extract from them practical benefit.

So the first step in solving the problem of AP should be a radical revision of its doctrine [10]. The basis of this phase of the work was done by the author of these lines over 30 years ago. The wellknown classical scientific materials was supplemented by the results of their own research $[11,12]$. A new perception of the causes and mechanisms of development of AP has identified the revision of the principles of treatment of such patients. The already obtained results of treatment of the severest forms of AP are a confirmation of this [13]. Already achieved results allowed to declare a guaranteed opportunity to prevent purulent complications of AP at certain stages of its development.

The purpose of this message is to draw the attention of a wide circle of specialists and researchers on the need for a radical change in the system of views on the nature of acute pneumonia. This is not only a primary and determining factor in solving the problem, but also the most difficult. We are talking about changing perceptions, which "Corresponding Author: Prof. Igor Klepikov, Pediatric Surgeon, 2116 27th St. NE Renton, WA, USA; E-mail: igor.klepikov@yahoo.com

Citation: Klepikov I (2017) The New Doctrine of Acute Pneumonia-the Key to Solve the Problem. Int J Pediatr Neonat Care 2: 124. doi: https://doi. org/10.15344/2455-2364/2017/124

Copyright: (c) 2017 Klepikov. This is an open-access article distributed under the terms of the Creative Commons Attribution License, which permits unrestricted use, distribution, and reproduction in any medium, provided the original author and source are credited. 
Citation: Klepikov I (2017) The New Doctrine of Acute Pneumonia-the Key to Solve the Problem. Int J Pediatr Neonat Care 2: 124. doi: https://doi. org/10.15344/2455-2364/2017/124

Page 2 of 5

evolved over the years and which has brought up not one generation of doctors. Without this transformation of ideas about the AP further efforts will be blind and not consciously. A series of lectures and seminars on the new doctrine AP can give the essential kinetics of this process. Now we only need awareness of the need of such work and its beginning.

\section{References}

1. https://www.thoracic.org/patients/patient-resources/breathing-in-america/ resources/chapter-15-pneumonia.pdf

2. World Health Organization (2009) New WHO/UNICEF action plan to tackle pneumonia. World Health Organization, Geneva, Switzerland.

3. Jain S, Williams DJ, Arnold SR, Ampofo K, Bramley AM, et al. (2015) Community-Acquired Pneumonia Requiring Hospitalization among U.S. Children. N Engl J Med 372: 835-845.

4. Elemraid MA, Thomas MF, Blain AP, Rushton SP, Spencer DA, et al. (2015) Risk factors for the development of pleural empyema in children. Pediatric Pulmonology 50: 721-726.

5. Klepikov I (2017) Acute Pneumonia in Children - Illness or Infection? Open Letter to the Editorial Staff. J Infect Non Infect Dis 3: 017

6. Klepikov I (2017) Acute Pneumonia in Children:The Price of Illusions and Delusions. Journal of Pediatric Care 3: 1-4

7. https://gehealthcloud.devpost.com/forum_topics/6433-tablet-againstpneumonia-modern-delusion-igor-klepikov-md-professor

8. Pabary R, Balfour-Lynn MI (2013) Complicated pneumonia in children. Breathe 9: 210-222

9. http://www.uptodate.com/contents/epidemiology-clinical-presentation-andevaluation-of-parapneumonic-effusion-and-empyema-in-children

10. Klepikov I (2017) Why Need a New Concept of Acute Pneumonia. J Tradit Med Clin Natur 6: 212

11. Klepikov I (2017) The Role of Cardiovascular Disorders in the Pathogenesis of Acute Pneumonia. J Cardiol \& Cardiovasc Ther 4: 555628.

12. Klepikov I (2017) The Meaning of Pulmonary Reflexes in the Pathogenesis of Acute Pneumonia. Intern Med 7: 232.

13. Klepikov I (1989) Acute pneumonia and its purulent and destructive complications in children in the midst of a major industrial centre of Western Siberia.Dissertation for Degree of Doctor of Medical Sciences.(in Russian,USSR) 